\title{
Dynamic Weight Overload Monitoring System Of Truck Based On Beidou Navigation Satellite System
}

\author{
Wei-gang PAN* \\ School of Information Science and Electronic Engineering, Shandong Jiaotong University, Jinan \\ 250357, China \\ panweigang1980@163.com
}

Keywords: beidou, dynamic weighing, often strain gauge.

Abstract. This paper is based on the Beidou satellite navigation system for heavy trucks weighing dynamic monitoring of a system overload, overload monitoring paper discusses the principle of overload monitoring systems, methods, and each unit of software and hardware components. Monitoring system to collect by pasting on a heavy-duty truck suspension strain gauge load data of the vehicle by $\mathrm{AD}$ analog to digital conversion process to the MCU information via CAN bus, MPU6050 to correct the inclination of the vehicle due to the load variation caused Beidou satellite module the position of vehicle, data collection can be saved to the system's EEPROM can be transmitted to the relevant regulatory authorities, to prevent overloading of trucks, vehicle driving safety effectively protected. Experimental tests proved that the strain gauge on the vehicle can have a load deformation when the measured vehicle load changes, via CAN bus data information is displayed on the LCD screen, satellite packets can be updated in real time, can be carried out on the vehicle monitoring system .

\section{The Principle Of Dynamic Weighing Overload Monitoring}

The acquisition is overloaded vehicle load monitoring system is an important content, only to obtain accurate data in order to load the vehicle overload monitoring, vehicle monitoring have substantive significance.

Analysis Of The Structure Of Heavy-Duty Truck. Here are many car bearing structure, load-bearing structure of trucks, mostly frame and elastic components, the force applied to the body more in the frame, the leaf springs to pass. Leaf spring is one of the important load-bearing structure of trucks. The leaf spring then acts on the body gravity attenuated frame, especially when the vehicle vibration can be effectively cushion the vehicle body force to disperse spring, reduce the impact on the frame. The following figure shows the frame and heavy-duty truck leaf spring suspension shown in Figure 1.

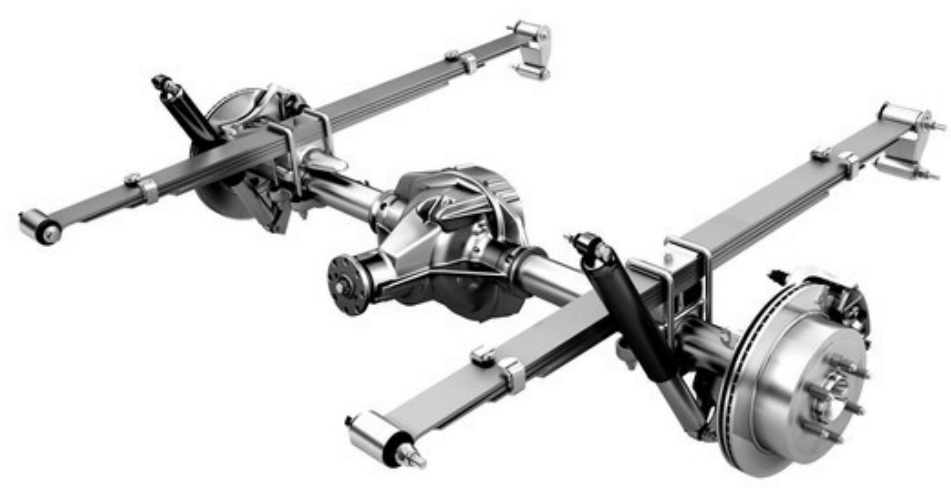

Fig.1 a schematic view of truck suspension

Overloading Of Lorries Weighing Method. By load measured using strain gauge, the strain gauge attached to the middle of the leaf spring when the truck load pressure spring, so that the leaf 
spring is deformed due to the strain gauge is deformed by the deformation of leaf spring, so that the voltage of the signal output terminal changes, high-precision $\mathrm{AD}$ voltage to a total acquisition via CAN bus controller MCU processing to send, and then calculate the total load.

\section{Beidou Overload Monitoring System Implementation}

Working Principle Of The System. Overload monitoring system by detecting deformation laden vehicle at the leaf springs, the deformation strain gauges into a voltage value, data collection terminal controller sends a data package by CAN controller sends data to the data processing terminal, truck load analysis, At the same time, the Beidou satellite navigation system will transmit location information to the vehicle and lorry load monitoring center, to achieve the truck load monitoring.

System Units. The data processing unit, a data acquisition unit, CAN communication module, the Beidou satellite communication module, plate deformation detection sensor, attitude sensor, communication cable.

The System Control Unit.M32F103RCT6 SCM data processing unit and the data acquisition controller uses STMicroelectronics has introduced the use of ARM Cortex-M3 32-bit microcontroller core, which reached 256K FLASH configuration, so storage space is more powerful, rich built peripherals, having CAN controllers, data communication convenience, the clock frequency of up to 72M, run fast. In the overload monitoring system has having high processing efficiency.

Beidou Satellite Communication Module. Beidou satellite navigation system, abbreviated BDS, independent research and development is being implemented in China, independent operation of global satellite navigation systems, GPS with the United States, Russia's GLONASS, the EU's Galileo system is compatible with the common global satellite navigation system, and said the four major satellite navigation system. In addition, the Beidou navigation system is an active two-way ranging two-dimensional navigation, ground control center system solver, three-dimensional positioning data for users, different from the traditional GPS passive individual pseudo-code Ranging three-dimensional navigation. The monitoring system used by the Beidou satellite navigation module, navigation terminal can support Beidou satellite navigation system to help locate navigation terminal, Beidou satellite navigation system is now beginning to enter the civilian phase.

CAN Communication Module. AN communication is in communication with the data acquisition system processing unit bridges, CAN controller with built-in CAN controller STM32, STM32 is built bxCAN, namely, the basic extended CAN. It supports the CAN protocol 2.0A and 2.0B. Its design goal is to minimize the load on the CPU to efficiently process a large number of the received packets. It also supports priority claim packets sent (priority feature is software configurable). For safety critical applications, bxCAN provide support for time-triggered communication mode all required hardware functionality. Supports CAN protocol 2.0A and 2.0B active mode, up to 28 variable filter group. And flexible configuration, easy to use.

CAN signal transmission also requires CAN transceiver, CAN transceiver as the interface between the CAN controller and the physical bus, and provides differential transmit and receive functions for the CAN controller. TJA1050 transceiver for the automotive industry, high-speed CAN application design, transfer rates up to $1 \mathrm{Mbit} / \mathrm{s}$.

CAN controller and CAN transceiver cooperate to stabilize the system transmits data to ensure the stability of the signal.

Orientation-Detecting Sensor. Posture detecting sensor is used MPU6050 acceleration angular velocity sensor, MPU6050 the integrated 6-axis motion processing components. Angular velocity full scale range of $\pm 250, \pm 500, \pm 1000$ and $\pm 2000^{\circ} / \mathrm{sec}$ (dps), can accurately track fast and slow movements, and the user can control the accelerator program full scale range of $\pm 2 \mathrm{~g}, \pm 4 \mathrm{~g} \pm 8 \mathrm{~g}$ and \pm 16 g. IIC interface module connected to the controller, using advanced digital filtering technology, can effectively reduce the measurement noise, improve the measurement accuracy through. With fusion algorithm, using a single acceleration or the angular velocity posture solver out solutions Dynamic Posture operator uses a gesture fusion algorithm. To ensure the stability and reliability of dynamic data. 
Beidou Overload Monitoring System Block Diagram

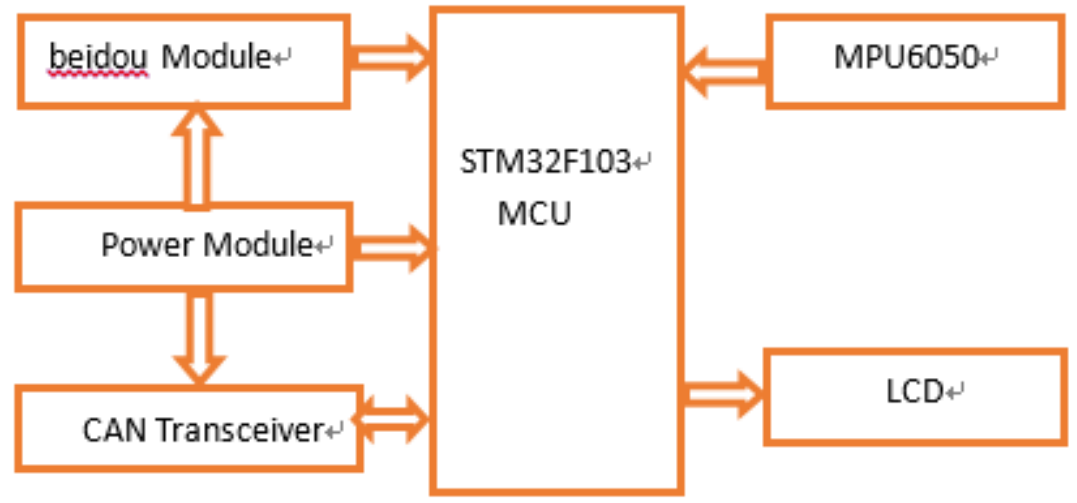

Fig.2 the data processing terminal

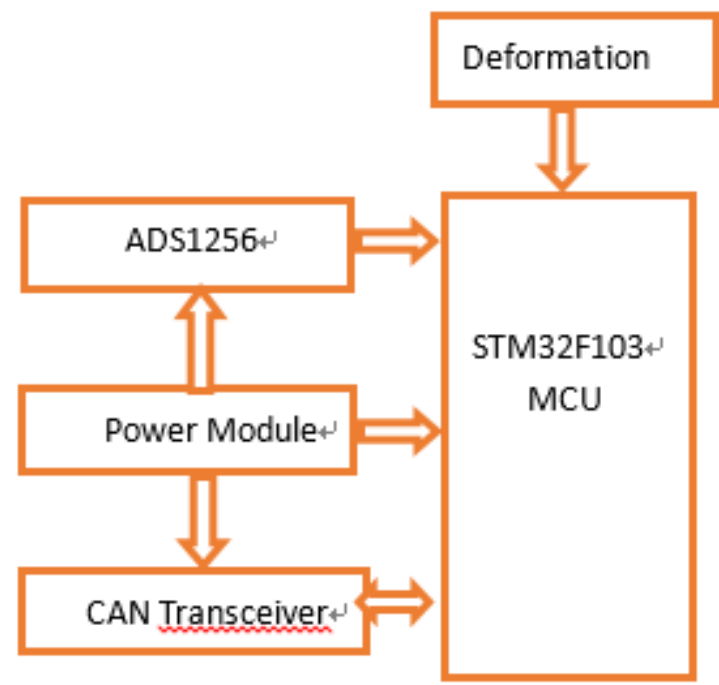

Fig.3 data acquisition unit

\section{Beidou Overload Monitoring System Hardware And Software Implementation}

\section{Working Schematic}

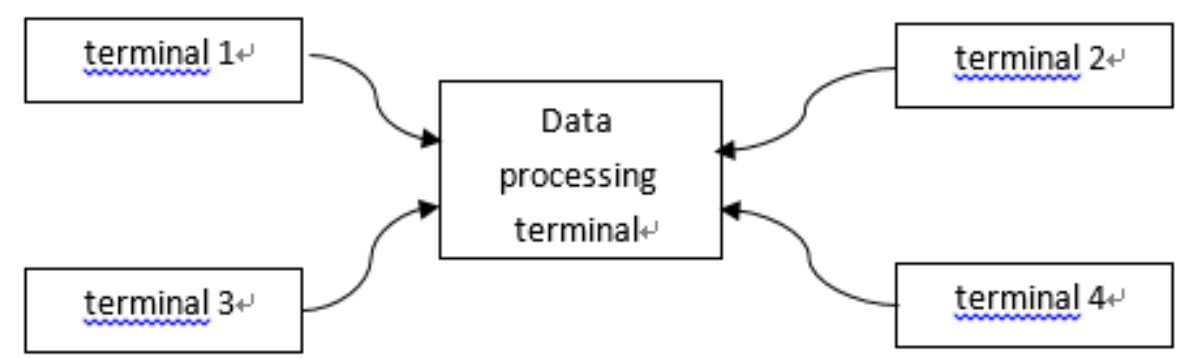

Fig.4 system work schematic composition 


\section{Work Flow Chart}

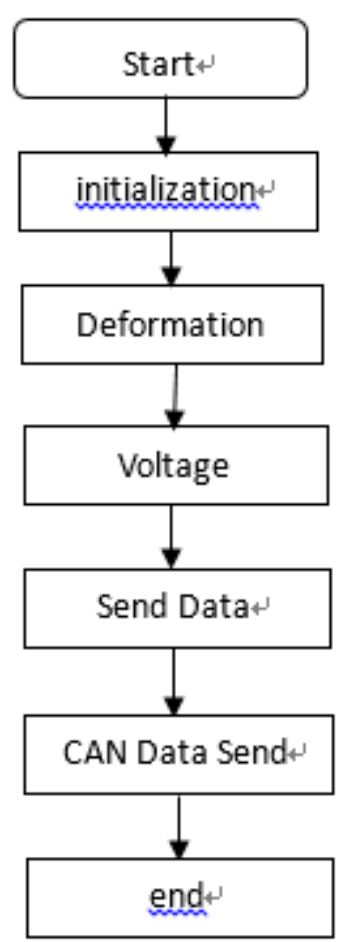

Fig. 5 flowchart data collection

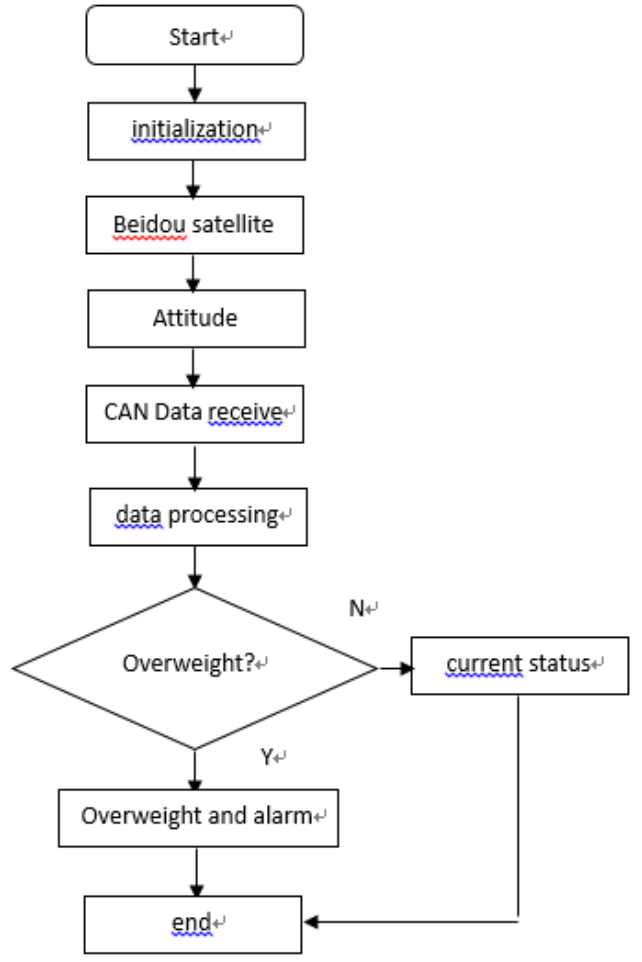

Fig.6 data processing work flow chart

Data Processing And Analysis. Data collection terminal by a leaf spring attached to the strain gauge, $\mathrm{AD}$ and MCU module composed of strain gauges attached to the leaf spring, the leaf spring on each group stick a strain gauge, when the vehicle load, the leaf spring is deformed, the strain gauge the deformation is converted to a voltage value, the output voltage of the strain gauge itself is very small, internally amplified output after AD, ADS1256 24-bit AD converter chip, high output accuracy and stability.

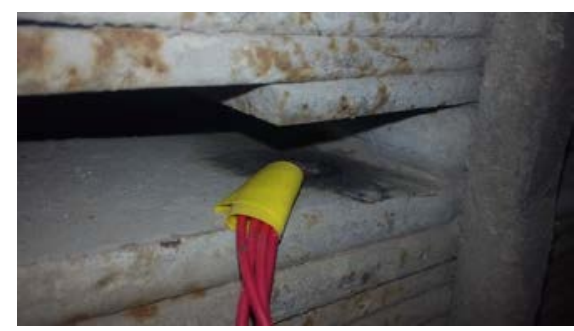

Fig.7 attached strain gages

Four collection terminal, is transferred to the data processing terminal voltage value, with the current vehicle attitude, comprehensive calculation load of the vehicle.

Tab.1 Acquisition System Voltage

\begin{tabular}{|l|c|c|c|c|}
\hline $\begin{array}{l}\text { Acquisition } \\
\text { System }\end{array}$ & Left Front & Right front & Left Rear & Right rear \\
\hline Voltage value (V) & 0.124 & 0.098 & 0.231 & 0.082 \\
\hline
\end{tabular}

Table 2 Load measurement data

\begin{tabular}{|c|c|c|c|c|}
\hline The actual load & $1100 \mathrm{KG}$ & $2205 \mathrm{KG}$ & $3150 \mathrm{KG}$ & $4050 \mathrm{KG}$ \\
\hline $\begin{array}{c}\text { Processing } \\
\text { voltage }\end{array}$ & 0.312 & 0.625 & 0.893 & 1.147 \\
\hline $\begin{array}{c}\text { Load } \\
\text { measurement }\end{array}$ & $1090 \mathrm{KG}$ & $2183 \mathrm{KG}$ & $3129 \mathrm{KG}$ & $4036 \mathrm{KG}$ \\
\hline
\end{tabular}

Inclination of the vehicle is calculated. Measured by the acceleration sensor voltage value after three axes RC filter, respectively $A_{x}, A_{y}, A_{z} \circ A_{x}, A_{y}$ In closing amount XOY plane to the horizontal 
plane of projection $\sqrt{\left(A_{x}-X_{0}\right)^{2}+\left(A_{y}-Y_{0}\right)^{2}} \cos \theta, A_{z}$ In the horizontal projection of $\left(A_{z}-Z_{0}\right) \sin \theta \quad$ since the resultant vector is zero, then $\sqrt{\left(A_{x}-X_{0}\right)^{2}+\left(A_{y}-Y_{0}\right)^{2}} \cos \theta=\left(A_{z}-Z_{0}\right) \sin \theta$ 。 The bevel angle is calculated as $\tan \theta=\frac{\sqrt{\left(A_{x}-X_{0}\right)^{2}+\left(A_{y}-Y_{0}\right)^{2}}}{A_{z}-Z_{0}}$, Wherein each represent $X_{0}, Y_{0}, Z_{0}$ axis conversion on the initial value.
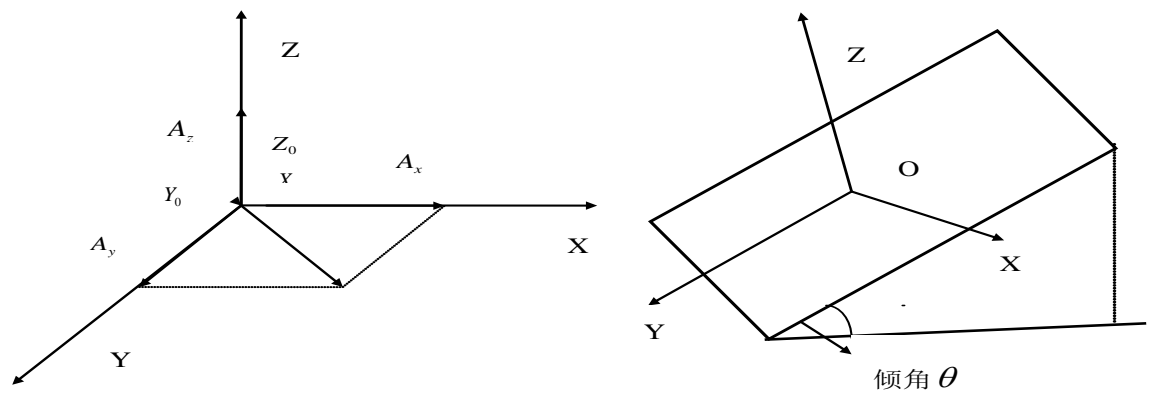

Fig.8 Schematic angle measurement

Tab.3 angle sensor data

\begin{tabular}{|c|c|c|c|c|}
\hline Reading times & the first time & the second time & the third time & the fourth time \\
\hline Reading angle & 8 & 6 & 11 & 5 \\
\hline
\end{tabular}

Experimental Results Verify. The test vehicle uses Fukuda light truck trucks rated load is small, the next picture shows the actual course of the experiment in the form of images and install.

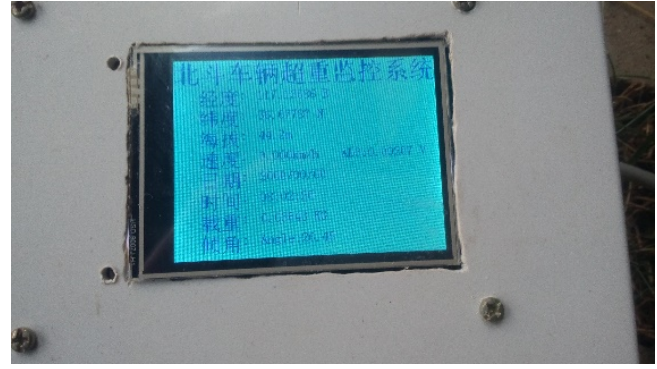

Fig.9 test voltage value displayed

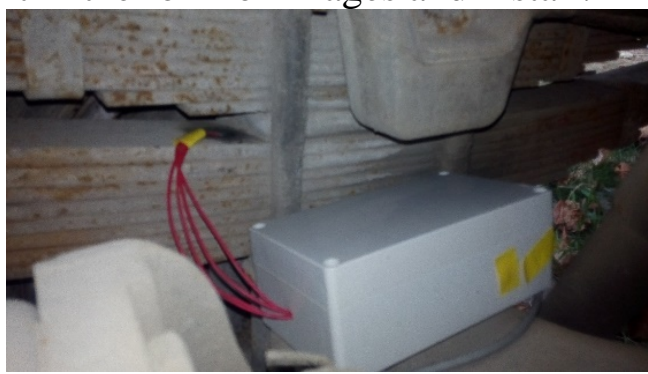

Fig.10 experimental installation image

\section{Conclusion}

This article is designed based on the success of the Big Dipper load vehicle dynamic weighing overload monitoring system to collect the vehicle by pasting on a heavy-duty truck suspension strain gauge load data, analog to digital conversion by AD to the MCU processing information via CAN bus, MPU6050 to correcting the inclination of the vehicle due to the load variation, the Beidou satellite positioning module to the vehicle's location data collection system can be saved to the EEPROM, it can be sent to the relevant regulatory authorities to prevent the overloading of trucks, vehicle driving safety effectively protected. Experimental tests proved that the strain gauge on the vehicle can have a load deformation when the measured vehicle load changes, via CAN bus data information is displayed on the LCD screen, satellite packets can be updated in real time, can be carried out on the vehicle monitoring system .

\section{Acknowledgements}

This work was financially supported by the Chinese Shandong Province Natural Science Fund Project(Number:ZR2013ZEM006), China Department of transportation Project 
(Number:2014329817130) and Department of transportation of Shandong province Chinese Project(Number:2013A16-04).

\section{References}

[1] M Ge, G Gendt, M Rothacher, C Shi, J Liu. Resolution of GPS carrier-phase ambiguities in Precise Point Positioning (PPP) with daily observations[J]. Journal of Geodesy, 2008, 82(7):389-399.

[2] L He, M Ge, J Wang. Experimental Study on the Precise Orbit Determination of the BeiDou Navigation Satellite System[J]. Sensor, 2013, 13(3):2911-2928.

[3] Zhou Zhi-Feng, Cai Ping, Chen Ri-Xing, Li Zhi-Gang. Method of processing data of weigh-in-motion of vehicles based on nonlinear curve-fitting.Journal of Shanghai Jiaotong University[J]. 2006,40(5):709-712.

[4] Meli E., Pugi L. Preliminary development, simulation and validation of a weigh in motion system for railway vehicles.Meccanica, 2013, 48(10): 2541-2565, December. 